\title{
A Dual Loop Control System For Dense Medium Coal Washing Processes With Sampled and Delayed Measurements
}

\author{
Lijun Zhang, Member, IEEE, Xiaohua Xia, Fellow, IEEE, and Bing Zhu
}

\begin{abstract}
A dual closed-loop control system is developed for a dense medium coal washing process. The controller is designed to improve the separation efficiency and energy efficiency of the dense medium cyclone coal beneficiation process simultaneously making use of a model predictive control (MPC) method and a pumped-storage system. In the dual loop control, the outer loop is designed for medium density profile optimization and the inner loop is used for actuator control of the medium supplying circuit, taking into consideration of the interplay of the energy efficiency and separation efficiency. Mathematical models of the pumpedstorage system and the magnetite recycle process of the dense medium circuit are developed and employed in the design of the advanced controller. The effectiveness of the designed control system is demonstrated by simulations based on operational data from a South African coal beneficiation plant.
\end{abstract}

Index Terms-Dense medium cyclone, coal washing, dualloop control, pumped-storage system, model predictive control, sampled \& delayed measurements.

\section{INTRODUCTION}

Cleaner utilization of coal is of vital importance because it is still the largest contributor to electricity generation [1]. This paper deals with coal cleaning processes that remove undesirable impurities from run-of-mine (ROM) coal to reduce pollutant emission and improve efficiency of coal utilization.

The most popular and productive coal cleaning technique is the dense medium cyclone (DMC) washing process that features high separation efficiency at high throughput [2].

Separation efficiency control of the DMC process used by industries today are based on either rule-of-thumb or empirical approaches. In previous work, an open loop controller making use of geological samples from the ROM was designed [3]. Measurement of the quality of fine coal from the output of the DMC was not assumed due to its high cost and long delay. In particular, fine coal obtained after the DMC process is usually sampled and sent to an off-site laboratory to analyze its compositions. The off-site measurement brings in delays of several hours. This delay, together with the sampled measurement, contribute negatively to the quality of coal obtained.

Introducing on-site measurement devices will help to reduce this measurement delay. It was found that the shortest time

Lijun Zhang and Xiaohua Xia are with the Department of Electrical, Electronic and Computer Engineering, University of Pretoria, Pretoria 0002, South Africa. Email: lijun.zhang@up.ac.za; xxia@up.ac.za. Bing Zhu is with the Seventh Research Division, Beihang University, Beijing 100191, P.R.China. He was with the Department of Electrical, Electronic and Computer Engineering, University of Pretoria, Pretoria 0002, South Africa. Email: zhubing@buaa.edu.cn required by measurement equipment available in market is five minutes according to [4].

The benefits of the measuring equipment must be studied because of the high cost. This however cannot be done without a properly designed controller capable of making full use of the measurement equipment. With this purpose, a closed-loop controller was designed in [5], making use of sampled and delayed measurements from the output of the DMC. However, the open loop controller [3] and the closed-loop controller [5] only deal with optimization of density profile of the medium used. A control system for the medium solution preparation process to track the optimized density profile was only studied by a more recent paper [6]. Moreover, the interactions between the DMC process and medium solution preparing process are ignored in the design of all the three controllers. Therefore, a complete design is needed to facilitate implementation of the required control. This is one of the main purposes of the controller design presented in this study.

In addition, the DMC cleaning processes are highly energy intensive because of the energy usage of the medium pumping system. Reducing energy consumption of the DMC process is an urgent task for coal mines. A pumped-storage system (PSS) was introduced in [7] to reduce energy waste due to over pumping in view of the successful application of demand side management (DSM) technologies to improve energy efficiency of industrial processes, such as domestic pumping systems [8], [7], [9], freight trains [10], [11], [12], [13], [14], [15], hybrid solar power systems [16], [17], [18], and mining processes [19], [20], [21], [22], [23], [24], [25], [26], etc. The PSS scheme, however, hasn't been considered by existing DMC controllers. Therefore, the other aim of the control system designed is to make use of the PSS scheme to improve energy efficiency of the DMC process.

In summary, this study aims to simultaneously improve the system's energy and separation efficiencies by a control system incorporating a pump storage system. This is achieved by a dual closed-loop control system using model predictive control (MPC) method (detailed in Section IV). Motivations of adopting MPC include its predictive control nature which helps to deal with the sampled and delayed measurements, its ability to cope with constraints in the design process, and its robustness against model plant mismatch and external disturbances that exist inevitably [27], [28]. This is further evidenced by the fact that MPC is one of the most used control method in industrial and commercial systems [29], [30]. In particular, application of MPC to address problems raised 


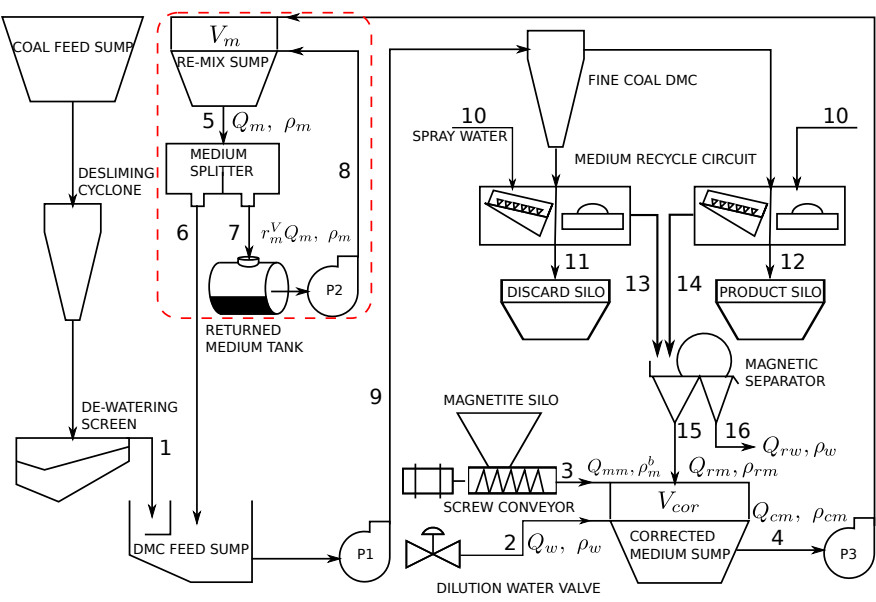

Fig. 1: DMC coal cleaning process with a PSS

from energy systems has been studied extensively with a great success. For instance, MPC was use in controlling of pumping systems [31], [8], solar power systems [16], economic power dispatching [32], [33], etc.

Although coal-to-medium ratio affects the separation efficiency of the DMC process [34], it is not considered in this study because the flow rate of medium supplied to the DMC is not affected by the PSS and controllers designed.

The rest of this paper includes four parts. Section II gives a brief introduction of the DMC process operating with a PSS. Section III presents a brief review of the DMC model developed in [35] and the detailed modeling process of the medium circulation process. Section IV discusses the controller design process and Section V presents some simulation results to validate the effectiveness of the proposed control approach. Section VI concludes the findings.

\section{The DMC CLEANING PROCESS WITH PSS}

Fig. 1 shows the schematic diagram of a DMC coal washing process operating with a PSS proposed in [7] (the part in the dotted box). The ROM coal is first processed by a de-slimming cyclone and de-watering screen, after which it is fed to the DMC feed sump where it gets blended with the dense medium. This mixed coal and medium slurry is then fed to the DMC for separation. In the DMC, the feed coal is separated into two streams, i.e. fine coal exiting at the top of the DMC and the discard exiting at the bottom of the DMC. These two slurries streams from the output of the DMC are subject to drain and rinse screens for magnetite recycle. The final outputs are stored in product silo and discard silo respectively for further processing. The diluted medium recovered are then collected and sent to a magnetic separator for magnetite recovery.

The recovered medium is in the form of concentrated magnetite and fed to a corrected medium sump, where extra magnetite and water are added to formulate the required medium solution. The valve used to add water and the screw conveyor used to add magnetite to the corrected medium sump are the actuators that can be controlled to adjust the density of the medium and therefore determines the fine coal quality.
In the current operation of the process studied, large amount of energy is wasted by the corrected medium pump because excessive amount of medium is pumped up from the corrected medium sump at the bottom to the top of the plant. The surplus medium pumped up is simply redirected by a medium splitter to flow back to the corrected medium sump without entering the separation process (see flow stream 7 in Fig. 1). In an energy audit carried out at the mine studied, it was identified that only $25 \%$ of the medium pumped is used in by the separation process. The $75 \%$ over pumping leads to poor energy efficiency and high energy cost for the plant.

In order to reduce energy consumption of the process without jeopardizing fine coal quality, a PSS consisting of an intermediate storage tank, a secondary pump and a re-mix sump was introduced in [7]. This PSS, shown in the dotted box in Fig. 1, forms a secondary medium circulation loop, which captures and pumps the excess medium returned by the medium splitter back to the top of the plant, preventing this portion of medium from flowing down to the bottom of the plant and therefore reducing energy consumption of the corrected medium pump. Although one more pump is added in the process, it can be observed that the differential head of the added pump is much shorter than that of the corrected medium pump, resulting in a pump with smaller size hence less energy consumption. With this structural change, energy efficiency of the plant can be improved remarkably as discussed in [7].

Consequently, the DMC coal preparation process with a PSS operates similar to the original configuration but with an extra medium circulation loop is obtained as shown in Fig. 1.

\section{Process modeling}

The DMC model developed in [35] is given here followed by detailed modeling of the medium circulation process.

\section{A. Model of the DMC}

The DMC model suitable for controller design was developed in [35]. A brief review of this model is provided here.

1) The DMC feed sump: With reference to Fig. 1, the percentages of ash, sulfur, moisture and volatile, $x_{i, c}$, and the percentage of the dense medium, $x_{i, m}$, in the slurry feeding to the DMC can be calculated by the following equations [35].

$$
\begin{gathered}
\dot{\rho}_{m b}=-\frac{Q_{m b}}{V_{m b}} \rho_{m b}+\frac{Q_{m}}{V_{m b}} \rho_{m}+\frac{1}{V_{m b}} W_{\text {ore }}, \\
x_{i, c}=\frac{x_{\text {ore },} W_{\text {ore }}}{Q_{m b} \rho_{m b}} \\
x_{i, m}=\frac{Q_{m} \rho_{m}}{Q_{m b} \rho_{m b}}
\end{gathered}
$$

where $\rho_{m b}$ is the density of the mixed slurry. $V_{m b}, Q_{m b, m}, \rho_{m}$ and $W_{\text {ore }}$ are, respectively, the volume of the DMC feed sump, the medium flow rate, the medium density, and the mass flow rate of coal feed to the DMC feed sump. $Q_{m b}$ is the flow rate of the mix to the DMC. $x_{\text {ore }, c}=\left[x_{\text {ore }, a s h}, x_{\text {ore }, S}, x_{\text {ore }, H_{2} O}, x_{\text {ore }, \text { vol }}\right]^{T}$ represents the percentages of the different compositions in the ROM coal. The subscript $c$ denoting a set with elements \{ash, sulfur, moisture, volatile $\}$ is used to simplify notations. 
2) The DMC: After mixed in the DMC feed sump, the slurry then enters the DMC where the separation occurs. Compositions of slurries that exit the DMC are governed by following equations derived from mass flow balance [35].

$$
\begin{aligned}
\dot{x}_{o, c}= & \frac{1}{V_{o} \rho_{o}}\left[W_{i} x_{i, c}-Q_{o} \rho_{o} x_{o, c}-Q_{u} \rho_{u} x_{u, c}-V_{o} x_{o, c} \dot{\rho}_{o}\right. \\
& \left.-V_{u} x_{u, c} \dot{\rho}_{u}-K_{u, c} V_{u} \rho_{u}\left(\rho_{c}-\rho_{m}\right)\left(x_{i, c}-x_{u, c}\right)\right], \\
\dot{x}_{u, c}= & \frac{1}{V_{u} \rho_{u}}\left[W_{i} x_{i, c}-Q_{o} \rho_{o} x_{o, c}-Q_{u} \rho_{u} x_{u, c}-V_{o} x_{o, c} \dot{\rho}_{o}\right. \\
& \left.-V_{u} x_{u, c} \dot{\rho}_{u}-K_{o, c} V_{o} \rho_{o}\left(\rho_{m}-\rho_{c}\right)\left(x_{i, c}-x_{o, c}\right)\right], \\
\dot{x}_{o, m}= & \frac{1}{V_{o} \rho_{o}}\left[W_{i} x_{i, m}-Q_{o} \rho_{o} x_{o, m}-Q_{u} \rho_{u} x_{u, m}-V_{o} x_{o, m} \dot{\rho}_{o}\right. \\
& \left.-V_{u} x_{u, m} \dot{\rho}_{u}-K_{u, m} V_{u} \rho_{u}\left(\rho_{o, m}-\rho_{m}\right)\left(x_{i, m}-x_{u, m}\right)\right], \\
\dot{x}_{u, m}= & \frac{1}{V_{u} \rho_{u}}\left[W_{i} x_{i, m}-Q_{o} \rho_{o} x_{o, m}-Q_{u} \rho_{u} x_{u, m}-V_{o} x_{o, m} \dot{\rho}_{o}\right. \\
& \left.-V_{u} x_{u, m} \dot{\rho}_{u}-K_{o, m} V_{o} \rho_{o}\left(\rho_{m}-\rho_{u, m}\right)\left(x_{i, m}-x_{o, m}\right)\right], \\
\dot{\rho}_{o}= & \frac{1}{V_{o}}\left[W_{i}-Q_{o} \rho_{o}-Q_{u} \rho_{u}-K_{u} V_{u}\left(\rho_{u}-\rho_{m}\right) x_{i, a s h}\right], \\
\dot{\rho}_{u}= & \frac{1}{V_{u}}\left[W_{i}-Q_{o} \rho_{o}-Q_{u} \rho_{u}-K_{o} V_{o}\left(\rho_{o}-\rho_{m}\right) x_{i, c}\right],
\end{aligned}
$$

where $x_{o, c}, x_{u, c}$ are $4 \times 1$ vectors representing the percentages of the compositions in the overflow and underflow of the cyclone, respectively. $x_{o, m}$ and $x_{u, m}$ are scalars that represent the percentage of magnetite in the overflow and underflow of the DMC, respectively. $\rho_{o}$ and $\rho_{u}$ are the densities of slurries exiting at the overflow and underflow of the cyclone. $W_{i}=Q_{m b} \rho_{m b}$ is the slurry feed rate to the cyclone. The volume parameter $V_{c}$ denotes the volume of slurry inside the cyclone which is assumed to split at a constant ratio $\alpha$ into the volume that reports to the overflow $V_{o}=\frac{\alpha}{1+\alpha} V_{c}$ and the volume that reports to the underflow $V_{u}=\frac{1}{1+\alpha} V_{c}$. Similarly, $Q_{o}$ and $Q_{u}$ are the flow rates to the overflow and underflow of the cyclone that are split by the same ratio $\alpha$. The $K_{o}, K_{u}, K_{o, c}, K_{u, c}, K_{o, m}$ and $K_{u, m}$ are DMC specific constants. $x_{i, C}$ is the percentage of carbon content in the DMC feed slurry, which is defined as $x_{i, C}=1-x_{i, m}-\sum_{c} x_{i, c}$.

The percentages of fixed carbon in the cyclone overflow and underflow after medium recycling process, $x_{o, C}$ and $x_{u, C}$, are obtained by

$$
\begin{aligned}
& x_{o, C}=1-\frac{1}{1-x_{o, m}} \sum_{c} x_{o, c}, \\
& x_{u, C}=1-\frac{1}{1-x_{u, m}} \sum_{c} x_{u, c} .
\end{aligned}
$$

The model given in equations (1)-(11) is derived from [35]. Advantages of this model are threefold. Firstly, the percentages of different components in the cyclone outputs can be obtained directly. Secondly, it only involves differential equations which takes much less time to solve compared to computational fluid dynamics based models. This makes it possible to apply controller designed with this model in a real-time environment. Thirdly, the accuracy of this model was validated with experiments to be more than $95 \%$, which is much better than that of empirical models used for control purposes [36].

\section{B. Model of the medium circulation process}

1) The magnetite recycle process: The medium trapped in the under- and over- flows of the DMC outputs are subject to drain and rinse screens for magnetite recycle. The diluted medium collected under the screens is then sent to magnetic separator for subsequent magnetite recovery as shown in Fig. 1. Studies have shown that in the DMC circuit, medium entering the DMC can only be recycled by a certain percentage because of losses in the circulation [37], therefore, the mass flow rate of concentrated magnetite from the output of the magnetic separator can be obtained by considering magnetite losses and mass flow balance of the magnetic separator. In the modeling process, the following assumptions are made:

1) $\beta$ percent of the magnetite entering the DMC will report to the magnetic separator for magnetite recovery;

2) $\eta_{s}$ percent of the magnetite entering the separator will be separated out from the diluted medium slurry;

3) The processing delay inside the magnetic separator is negligible; and

4) The concentrated magnetite from the outlet of the magnetic separator has a stable density, $\rho_{r m}$.

With the above assumptions, one can model magnetite recycle process and obtain the following equaton

$$
Q_{m}^{p}=\frac{Q_{c m}\left(\rho_{m}-\rho_{w}\right)}{\rho_{m}^{p}-\rho_{w}},
$$

where $Q_{m}^{p}$ and $\rho_{m}^{p}$ represent the volume flow rate and density of the magnetite particles carried in the dense medium. $Q_{w}$ and $\rho_{w}$ represent the volume flow rate and density of water contained in the medium solution. $Q_{c m}=\left(1-r_{m}^{V}\right) Q_{m}$ is the volume flow rate of the medium flowing into the DMC process, in which $r_{m}^{V}$ is the ratio at which the corrected medium is returned by the medium splitter (See Fig. 1).

Therefore, the mass flow rate of magnetite reporting to the magnetic separator can be obtained by

$$
m_{m}=\beta Q_{m}^{p} \rho_{m}^{p}=\frac{\beta \rho_{m}^{p} Q_{c m}\left(\rho_{m}-\rho_{w}\right)}{\rho_{m}^{p}-\rho_{w}},
$$

where $m_{m}$ is the mass flow rate of the magnetite reporting to the magnetic separator according to assumption 1 above.

Following equation (13) and assumptions 2-4, the volume flow rate of the concentrated medium can be obtained as

$$
Q_{r m}=\frac{\eta_{s} \beta Q_{c m} \rho_{m}^{p}\left(\rho_{m}-\rho_{w}\right)}{\rho_{r m}\left(\rho_{m}^{p}-\rho_{w}\right)} .
$$

2) The PSS: The densities of slurry flows in the PSS scheme must be modeled to facilitate controller design.

According to the mass balance of the re-mix sump, the density of medium that enters the DMC feed sump and DMC thereafter can be modeled by the following equation:

$$
V_{m} \frac{d \rho_{m}}{d t}=-Q_{m} \rho_{m}+r_{m}^{V} Q_{m} \rho_{m}+Q_{c m} \rho_{c m},
$$

where $\rho_{c m}$ is the density of corrected medium in the corrected medium sump, and $V_{m}$ is the volume of the re-mix box.

The density $\rho_{c m}$ is adjusted by controlling the volume flow rates of the water, $Q_{w}$, and the magnetite, $Q_{m m}$, to the corrected 


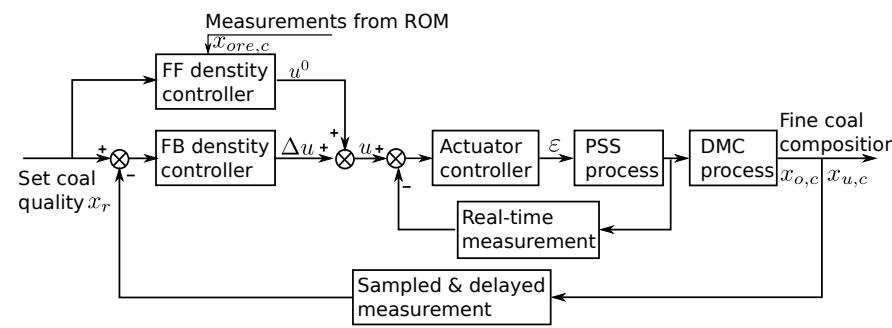

Fig. 2: Diagram of the dual-loop controller

medium sump. The density of the corrected medium can be obtained using

$$
V_{c o r} \frac{d \rho_{c m}}{d t}=-Q_{c m} \rho_{c m}+Q_{r m} \rho_{r m}+Q_{w} \rho_{w}+Q_{m m} \rho_{m}^{p},
$$

where $V_{c o r}$ is the medium volume in the corrected medium sump, $Q_{r m}$ and $\rho_{r m}$ are the flow rate and density of the magnetite recovered by the magnetic separator (flow stream 15 in Fig. 1). $Q_{m m}$ is the flow rate of the make-up magnetite particles added by the screw conveyor. The volume change of the medium in the corrected medium sump is described by

$$
\frac{d V_{c o r}}{d t}=Q_{r m}+Q_{w}+Q_{m m}-Q_{c m}
$$

The volume flow rate of the linear valve is a function of the fluid density and valve position:

$$
Q_{w}=C_{w} l_{w} \sqrt{\frac{\Delta P}{\rho_{w}}},
$$

where $C_{w}$ is the valve coefficient, $l_{w}$ is the valve position in percentage, and $\Delta P$ is the pressure drop across the valve.

The volume flow rate of the magnetite particles added by the screw conveyor can be related to the speed of the motor driving the conveyor belt by the following equation: ${ }^{1}$

$$
Q_{m m}=Q_{1} \frac{\rho_{m}^{b}}{\rho_{m}^{p}} N
$$

where $Q_{1}$ is a conveyor belt related constant, $\rho_{m}^{b}$ is the bulk density of the magnetite and $N$ is the motor speed in rpm.

\section{Controller DESIGN}

\section{A. Outer loop controller}

For the outer loop, sampled measurements on the percentages of ore compositions from different geological locations of the mine pits are used as feed forward information to derive a feed forward controller as shown in [3]. With help of the feed forward control, a closed-loop controller can be designed with sampled and delayed feedback measurements from the output of the DMC. Because the DMC process model is nonlinear with strong coupling between different states, a nonlinear model predictive controller is proposed to control the relative density of the medium. Diagram of the overall control system is depicted in Fig. 2, where the dual-loop control is clearly shown. In this figure, FF density controller

\footnotetext{
${ }^{1}$ Conveyor Eng. \& Mfg. Co. Screw conveyor components \& design. Version 2.20 .
}

and FB density controller refer to the feed forward control and feedback control of the outer loop controller, respectively.

To facilitate controller design, the DMC model presented in Section III-A is discretized using the Runge-Kutta method to be as follows [38]

$$
x(k+1)=f(x(k), u(k)),
$$

where the state of the DMC process at time $k T_{s}$ is $x(k)=$ $\left[x_{o, c}^{T}(k), x_{u, c}^{T}(k), x_{o, m}(k), x_{u, m}(k), \rho_{o}(k), \rho_{u}(k)\right]^{T}$, in which $T_{s}$ is the sampling period. The control variable is $u(k)=\rho_{m}(k)$.

1) Feed forward control: The primary goal of the feed forward control is to improve the quality of the coal. Reducing energy consumption of the DMC process by minimizing the density of medium circulating in the plant is treated as the secondary objective of the controller. As a result, the following objective function is adopted.

$$
J=\sum_{k=1}^{T / T_{s}}\left[k_{p}\left(x_{o, C}(k)-x_{r}(k)\right)^{2}+k_{e} u(k)^{2}\right]
$$

where $x_{r}(k)$ is the desired percentage of fixed carbon in the fines at sampling instant $k T_{s}, T$ is the operating time of the DMC circuit. For simplicity, $T$ is a multiple of $T_{s}$. Weights $k_{q}$ and $k_{e}$ are used to tune the controller and are chosen by users according to their preferences.

Physical and operational constraints of the DMC process are described by the following inequalities:

$$
\begin{gathered}
0 \leq x_{o, c}(k) \leq 1, \\
0 \leq x_{u, c}(k) \leq 1, \\
0 \leq x_{o, C}(k) \leq 1, \\
0 \leq x_{u, C}(k) \leq 1, \\
\left|\rho_{m}(k)-\rho_{m}(k-1)\right| \leq \Delta \rho_{m}, \\
\rho_{m}^{l} \leq \rho_{m}(k) \leq \rho_{m}^{u} .
\end{gathered}
$$

Inequalities (22), (23), (24), and (25) naturally exist as the percentages are from $0 \%$ to $100 \%$. Constraints (26) and (27) represent the limits on the rate of change and range of the medium density. $\Delta \rho_{m}, \rho_{m}^{l}$ and $\rho_{m}^{u}$ are, respectively, the limits on the rate of change, and the lower and upper limits of the density of the medium.

Define $U^{0}=\left[u^{0}(k), u^{0}(k+1), \cdots, u^{0}\left(k+T / T_{s}\right)\right]^{T}$. The feed forward controller solves the nonlinear optimization problem:

$$
U^{0^{*}}=\arg \min _{U^{0}} J
$$

subject to (20) and (22)-(27), whenever new measurement from the ROM is available.

Remark 1: According to the physical properties of the entire system, a feasible $u^{0}$ always exists to satisfy (20) and (22)-(27). In this section, the optimization (28) is an open loop one, and $u^{0}$ is subject to constraint (27), indicating that the reference $u^{0 *}$ (generated for the closed-loop MPC to track) is always feasible and bounded. 
2) Closed-loop MPC: Denote the DMC state and control reached by the feed forward controller as $x^{0}$ and $u^{0}$, and the carbon content in the fines as $x_{o, C}^{0}$. The closed-loop MPC is to maintain the carbon content making use of feedback information $x$ by introducing $\Delta u$ change to $u^{0}$ in order to compensate model plant mismatch and disturbances. Therefore, the objective function for the closed-loop MPC is derived from (21) and given as

$$
\begin{aligned}
J_{o}(k)= & \sum_{i=1}^{N_{p}}\left(k_{p} \Delta x_{o, C}^{2}(k+i \mid k)+k_{e} \Delta u(k+i-1 \mid k)^{2}\right. \\
& \left.+2 k_{p}\left[x_{o, C}^{0}(k+i)-x_{r}(k+i)\right] \Delta x_{o, C}(k+i \mid k)\right) \\
& +\sum_{i=1}^{N_{C}} 2 k_{e} u^{0}(k+i-1) \Delta u(k+i-1 \mid k),
\end{aligned}
$$

where $N_{p}$ and $N_{C}$ are the optimization and control horizons, $k$ denotes the current time $k T_{s}, \mid k$ means that the predicted value is based on the information up to $t=k T_{s}$, and $\Delta x_{o, C}=$ $x_{o, C}-x_{o, C}^{0}$ is the change of carbon content because of $\Delta u$.

The constraints (22)-(27) are transformed into

$$
\Omega\left(\Delta u(k \mid k), \ldots, \Delta u\left(k+N_{c}-1 \mid k\right)\right) \leq \gamma,
$$

where the nonlinear function $\Omega(\cdot)$ represents the constraints (22)-(27) and $\gamma$ represents the limits derived therein. The following terminal constraint is added to ensure the stability of the controller:

$$
\Delta x_{o, c}\left(k+N_{c} \mid k\right)=0 .
$$

Define $\Delta U(k)=\left[\Delta u(k \mid k), \Delta u(k+1 \mid k), \cdots, \Delta u\left(k+N_{c} \mid k\right)\right]^{T}$. The optimization of MPC can then be formulated by

$$
\Delta U^{*}(k)=\arg \min _{\Delta U(k)} J_{o}(k),
$$

subject to (20) and (30). The optimal control is implemented in a receding horizon scheme:

$$
\Delta u(k)=\Delta u^{*}(k \mid k),
$$

where $\Delta u^{*}(k \mid k)$ is the first entry of $\Delta U^{*}(k)$.

The final optimal medium density obtained by the closedloop controller is given by: $u=u^{0}+\Delta u$.

\section{B. Inner loop controller}

The aim of the inner loop controller is to track the optimal density profile set by the outer loop controller by manipulating the water addition valve and the magnetite addition conveyor. This essentially requires designing a controller for the PSS circuit. To achieve this, the model shown in Section III-B2 is first transformed into the following state space model.

Denote the state of the PSS by $\zeta=\left[\dot{V}_{c o r}, V_{c o r}, \dot{\rho}_{c m}, \rho_{c m}, \rho_{m}\right]^{T}$, and the control variables by $\varepsilon=\left[\dot{i}_{w}, \dot{N}\right]^{T}$, one can get the following equations from (15)-(19):

$$
\dot{\zeta}_{1}=\frac{\eta_{s} \beta \rho_{m}^{p}\left(1-r_{m}^{V}\right)^{2} Q_{m}^{2}}{V_{m} \rho_{r m}\left(\rho_{m}^{p}-\rho_{w}\right)}\left(\zeta_{4}-\zeta_{5}\right)+C_{w} \sqrt{\frac{\Delta P}{\rho_{w}}} \varepsilon_{1}+\frac{\rho_{m}^{b}}{\rho_{m}^{p}} Q_{1} \varepsilon_{2},
$$

$\dot{\zeta}_{2}=\zeta_{1}$

$$
\begin{aligned}
\dot{\zeta}_{3}= & \frac{1}{\zeta_{2}}\left[-\left(1-r_{m}^{V}\right) Q_{m} \zeta_{3}+\frac{\eta_{s} \beta \rho_{m}^{p}\left(1-r_{m}^{V}\right)^{2} Q_{m}^{2}}{V_{m}\left(\rho_{m}^{p}-\rho_{w}\right)}\left(\zeta_{4}-\zeta_{5}\right)\right. \\
& \left.-\zeta_{1} \zeta_{3}+C_{w} \sqrt{\Delta P \rho_{w}} \varepsilon_{1}+Q_{1} \rho_{m}^{b} \varepsilon_{2}\right] \\
\dot{\zeta}_{4}= & \zeta_{3}, \\
\dot{\zeta}_{5}= & \frac{\left(1-r_{m}^{V}\right) Q_{m}}{V_{m}}\left(\zeta_{4}-\zeta_{5}\right) .
\end{aligned}
$$

Take $\phi=\left[\phi_{1}, \phi_{2}\right]^{T}=\left[\zeta_{2}, \zeta_{5}\right]^{T}$ as the output, these equations can be decoupled and linearized using state feedback [39], [40].

Introduce the coordinate transformation $z=T(\zeta)=$ $\left[\begin{array}{lllll}\phi_{1} & \dot{\phi}_{1} & \phi_{2} & \dot{\phi}_{2} & \ddot{\phi}_{2}\end{array}\right]^{T}$, the above nonlinear equations (34)(38) can be transformed into a new linear system in the $z$ coordination as follows:

$$
\begin{aligned}
\dot{z} & =A z+B v, \\
\phi & =C z,
\end{aligned}
$$

where $\quad A=\left[\begin{array}{ccccc}0 & 1 & 0 & 0 & 0 \\ 0 & 0 & 0 & 0 & 0 \\ 0 & 0 & 0 & 1 & 0 \\ 0 & 0 & 0 & 0 & 1 \\ 0 & 0 & 0 & 0 & 0\end{array}\right], \quad B=\left[\begin{array}{ll}0 & 0 \\ 1 & 0 \\ 0 & 0 \\ 0 & 0 \\ 0 & 1\end{array}\right]$, $C=\left[\begin{array}{lllll}1 & 0 & 0 & 0 & 0 \\ 0 & 0 & 1 & 0 & 0\end{array}\right] . \quad v$ is the new control variable defined by

$$
v=F(\zeta)+T \varepsilon,
$$

where $\quad T=\left[\begin{array}{cc}C_{w} \sqrt{\frac{\Delta P}{\rho_{w}}} & \frac{\rho_{m}^{b}}{\rho_{m}^{p}} \\ \frac{\Theta_{m} C_{w}}{\zeta_{2}} \sqrt{\Delta P \rho_{w}} & \frac{\Theta_{m} \rho_{m}^{m} Q_{1}}{\zeta_{2}}\end{array}\right], \quad F(\zeta)=$ $\left[\left(\Theta_{m}^{3}+\frac{\Theta_{m} \Theta \rho_{r m}}{\zeta_{2}}\right)\left(\zeta_{4}-\zeta_{5}\right)-\Theta_{m}^{2} \zeta_{3}-\frac{\Theta_{m}}{\zeta_{2}}\left(Q_{c m} \zeta_{3}+\zeta_{1} \zeta_{3}\right)\right]$, in which $\Theta=\frac{\eta_{s} \beta \rho_{m}^{p}\left(1-r_{m}^{V}\right)^{2} Q_{m}^{2}}{V_{m} \rho_{r m}\left(\rho_{m}^{p}-\rho_{w}\right)}$ and $\Theta_{m}=\frac{\left(1-r_{m}^{V}\right) Q_{m}}{V_{m}}$.

The new linear system showing in equation (39) is controllable [39]. Therefore, the original nonlinear system can be controlled by designing the new control variable $v$. The original control variable $\varepsilon$ can be determined by

$$
\varepsilon=T^{-1}(-F(\zeta)+v) .
$$

The linearized model is further discretized to be

$$
\begin{aligned}
& z(k+1)=A_{d} z(k)+B_{d} v(k), \\
& \phi(k)=C_{d} z(k),
\end{aligned}
$$

where $A_{d}, B_{d}$ and $C_{d}$ and matrices obtained by applying zero-order holder method with sampling period of $T_{s}^{i n}$ to the continues model (39).

Based on (42), an MPC controller with the following stage objective function is designed for the inner loop:

$$
J^{i n}=\sum_{i=1}^{N_{p}^{i n}} e(k+i \mid k)^{T}\left[\begin{array}{cc}
k_{v} & 0 \\
0 & k_{d}
\end{array}\right] e(k+i \mid k)+\sum_{j=0}^{N_{c}^{i n}-1} k_{c} \varepsilon(k+j \mid k)^{2},
$$

where $e(k+i \mid k)=[\phi(k+i \mid k)-r(k+i \mid k)]$ is the tracking error, $k_{v}, k_{d}$ and $k_{c}$ are weighting factors, $N_{p}^{i n}$ and $N_{c}^{i n}$ are the predictive horizon and control horizon of the inner loop MPC, respectively. $r(k+i \mid k)=\left[\begin{array}{c}V_{\text {set }} \\ u(k+i-1 \mid k)\end{array}\right]$ is the vector 
consisting of the set volume of medium in the corrected medium sump and density set by the outer loop controller. It is noticed that the relationship, $N_{p}^{i n} T_{s}^{i n} \leq N_{c} T_{s}$, should hold all the time because the optimal density profile set by the outer loop control is defined over the interval $\left[k T_{s},\left(k+N_{c}-1\right) T_{s}\right]$.

The constraints are that of the upper and lower limits of the state variable and the control variable:

$$
\begin{aligned}
& \zeta_{\min } \leq \zeta(k) \leq \zeta_{\max } \\
& \varepsilon_{\min } \leq \varepsilon(k) \leq \varepsilon_{\max }
\end{aligned}
$$

where the subscript min and max denote the lower and upper limit, respectively. The following terminal constraint is added to guarantee the closed-loop stability of the inner loop system:

$$
e\left(k+N_{c}^{i n} \mid k\right)=0 .
$$

Define $\bar{\varepsilon}=\left[\varepsilon(k \mid k), \varepsilon(k+1 \mid k), \cdots, \varepsilon\left(k+N_{c}^{i n} \mid k\right)\right]^{T}$. The optimization in the inner loop MPC can be calculated by

$$
\bar{\varepsilon}^{*}(k)=\arg \min _{\bar{\varepsilon}(k)} J^{i n}(k),
$$

subject to (44) and (45).

\section{Stability analysis of the closed-loop system}

Assumption 1: For a large enough predictive horizon $N_{p}^{i n}$ and control horizon $N_{c}^{i n}$, there always exist an initial feasible solution to the optimization problem (46) at $k=0$.

Remark 2: The above assumption of initial feasibility is based on the fact that the system under consideration is practical, and there should be at least a feasible control $u$ satisfying all constraints, such that the closed-loop inner control system is stabilized.

As can be seen from the optimization (46), the inner loop MPC is designed in a standard framework of MPC with equality terminal constraint. It then follows that, if the optimization (46) problem is feasible initially (as given in Assumption 1), then it is feasible recursively [41]. The stability of the inner loop system is guaranteed by the recursive feasibility of the optimization. Convergence rate of the inner loop system can be tuned by $k_{v}, k_{d}, k_{c}$ and $N_{c}^{i n}$, such that the time-scale of the inner loop system can be assigned much faster than the outer loop system. It then follows that, in the time scale of the outer loop system, the inner loop system can be approximately regarded as an identity matrix between its control inputs (which are the rate of valve $\dot{l}_{w}$ and the rate of motor speed $\dot{N}$ ) and its output ( $V_{\text {cor }}$ and $\rho_{m}$, which are the input of the outer loop system).

Remark 3: According to time-scale separation [42] and singular perturbation [43], the transient process of the inner loop system should be tuned four times faster than that of the outer loop system, otherwise the overall stability is not necessarily guaranteed.

For the outer loop system, if there is no disturbance or measurement delay, the nominal system would progress as predicted, and its closed-loop system would be exponentially stable, which is ensured by the standard theory of nonlinear MPC with terminal equality constraint [44]. Disturbances and measurement delays can be viewed as accumulative perturbations, and they can be regarded to be rectified whenever the measurement is available. The accumulative perturbations are bounded, since measurements are available at every finite time. Bounds of the accumulative perturbations increase as time interval of the measurements grows. It then follows that the outer loop system is an exponentially stable system plus bounded perturbations, which is ultimately bounded according to Lemma 4.6 in [43]. The magnitudes of the ultimate bounds relate directly to the bounded perturbations.

The entire closed-loop system (combination of the outer loop and the inner loop systems) can be guaranteed ultimately bounded by carefully setting the control parameters, such that the time scale of the inner loop system significantly faster than that of the outer loop system. Correlation of the ultimate bounds of the entire system with disturbances and measurement delays is displayed in the section of simulation.

The above analysis is summarized by the following proposition.

Proposition 1: Consider the model of DMC and medium circulation process given by Section III, and suppose that Assumption 1 is satisfied. The controller is designed by combining the feedforward control (21)-(28), the outer loop control (29)-(33), and the inner loop control (43)-(46). It then follows that the overall system is ultimately bounded with respect to disturbances and measurement delays, and the ultimate bounds can be tuned by control parameters.

\section{Simulation}

Simulations based on a South African coal processing plant are given to verify the effectiveness the controller designed.

\section{A. Simulation configuration}

In simulations, 15 coal washing DMC modules run 16 hours per day. The coal feed rate to the DMC circuit is $W_{\text {ore }}=9.42 \mathrm{~kg} / \mathrm{s} . T_{s}=14 \mathrm{~s}$ is used to discretize the DMC model for the outer loop control and $T_{s}^{i n}=0.01 \mathrm{~s}$ is used to discretize the PSS circuit model for the inner loop control. Parameters of the DMCs are shown in Table I.

\section{B. The benchmark}

The designed control system is compared to the benchmark - the open loop feed forward control proposed in [3]. This choice of baseline is in accordance with the principles of energy efficiency measurement and verification [45].

In the following comparison, the tracking error of the set carbon percentage in the fine coal product is used as the separation efficiency indicator and the daily energy consumption of the 15 DMC modules is used as the energy efficiency indicator. In particular, the energy consumption of the benchmark is calculated by $E_{B}=15 \int_{0}^{T} \frac{u(t) Q_{m} g h}{3600000 \eta_{p}} d t$, where $T$ is the daily operation time, $g$ is gravitational acceleration, $h$ is the differential head of the corrected medium pump, and $\eta_{p}$ is the combined efficiency of the corrected medium pumping system. The number 3600000 is a unit conversion factor. The daily energy consumption of the plant with the proposed DCLC is calculated by $E_{D C L C}=15 \int_{0}^{T}\left(\frac{\rho_{c m}(t) Q_{c m} g h+u(t) r_{m}^{V} Q_{m} g h_{1}}{3600000 \eta_{p}}\right) d t$, where $h_{1}$ is the differential head of the secondary pump severing the PSS. $h=60 \mathrm{~m}, h_{1}=20 \mathrm{~m}, \eta_{p}=0.8$ and $g=9.8$ $\mathrm{m} / \mathrm{s}^{2}$ are used in the following calculations. 
TABLE I: Model parameters

\begin{tabular}{lll}
\hline Variable & Value & Unit \\
\hline$V_{m b}$ & 0.16 & $\mathrm{~m}^{3}$ \\
$Q_{m b, m}$ & 0.495 & $\mathrm{~m}^{3} / \mathrm{s}$ \\
$Q_{m}$ & 0.500 & $\mathrm{~m}^{3} / \mathrm{s}$ \\
$\alpha$ & 2 & - \\
$V_{c}$ & 0.38 & $\mathrm{~m}^{3}$ \\
$K_{o}$ & 0.22 & $\mathrm{~m}^{2} \mathrm{~s}$ \\
$K_{u}$ & 0.22 & $\mathrm{~m}^{2} \mathrm{~s}$ \\
$K_{o, c}$ & {$[2.00,3.90,1.50,8.90]^{T}$} & $\times 10^{-4} \mathrm{~m}^{3} / \mathrm{kgs}$ \\
$K_{o, m}$ & $4.80 \times 10^{-2}$ & $\mathrm{~m}^{3} / \mathrm{kgs}^{-4}$ \\
$K_{u, c}$ & {$[0.77,3.90,0.30,8.90]^{T}$} & $\times 10^{-4} \mathrm{~m}^{3} / \mathrm{kgs}$ \\
$K_{u, m}$ & $3.90 \times 10^{-2}$ & $\mathrm{~m}^{3} / \mathrm{kgs}$ \\
$\rho_{a s h}$ & 2000 & $\mathrm{~kg} / \mathrm{m}^{3}$ \\
$\rho_{S}$ & 1920 & $\mathrm{~kg} / \mathrm{m}^{3}$ \\
$\rho_{H_{2} O}$ & 1000 & $\mathrm{~kg} / \mathrm{m}^{3}$ \\
$\rho_{v o l}$ & 1100 & $\mathrm{~kg} / \mathrm{m}^{3}$ \\
$\rho_{m}^{b}$ & 2850 & $\mathrm{~kg} / \mathrm{m}^{3}$ \\
$\rho_{m}^{p}$ & 3500 & $\mathrm{~kg} / \mathrm{m}^{3}$ \\
$r_{m}^{V}$ & 0.75 & - \\
$\beta$ & 0.97 & - \\
$\eta_{s}$ & 0.98 & - \\
$V_{c o r}$ & 2 & $\mathrm{~m}^{3}$ \\
$V_{m}$ & 1 & $\mathrm{~m}$ \\
$C_{w}$ & 0.0002 & $\mathrm{~m}^{3} / \mathrm{h} \cdot \mathrm{Pa}$ \\
$\Delta P$ & 100 & $\mathrm{kPa}$ \\
$Q_{1}$ & $1.18 \times 10^{-5}$ & $\mathrm{~m}{ }^{3} / \mathrm{s}$ \\
\hline & &
\end{tabular}
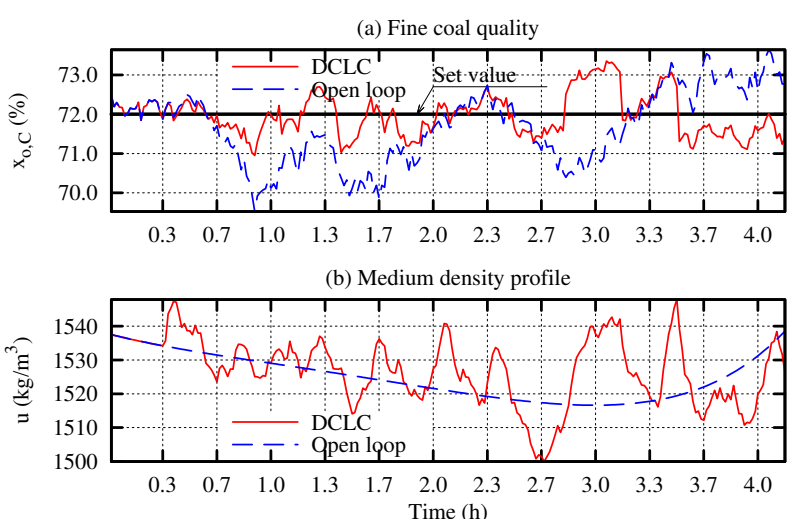

Fig. 3: DCLC v.s. benchmark: the outer loop control

\section{The dual closed-loop control}

In addition to parameters provided in Section V-A, the predicting and control horizons of the outer loop and inner loop controllers are set as $N_{p}=7, N_{c}=5, N_{p}^{i n}=8$, and $N_{c}^{i n}=4$.

1) Comparing the DCLC to the benchmark: Taking into account modeling inaccuracies and process disturbances, the performance of the controllers are compared under a 5\% random disturbance/uncertainty (Refer to [5] for more details).

Since the disturbances introduced are of random nature, average performance indicators of 20 simulation runs of the DMC with the designed control system and the benchmark controller are compared to provide a fair comparison.

Figs. 3-4 show the comparison between the DCLC and the benchmark control subject to $5 \%$ disturbance in case of 20 minute of measurement delay. Fig. 3 presents the average fine coal quality indicator and the medium density profile obtained by the DCLC and the benchmark controller, respectively. One (a) Medium density profile tracking
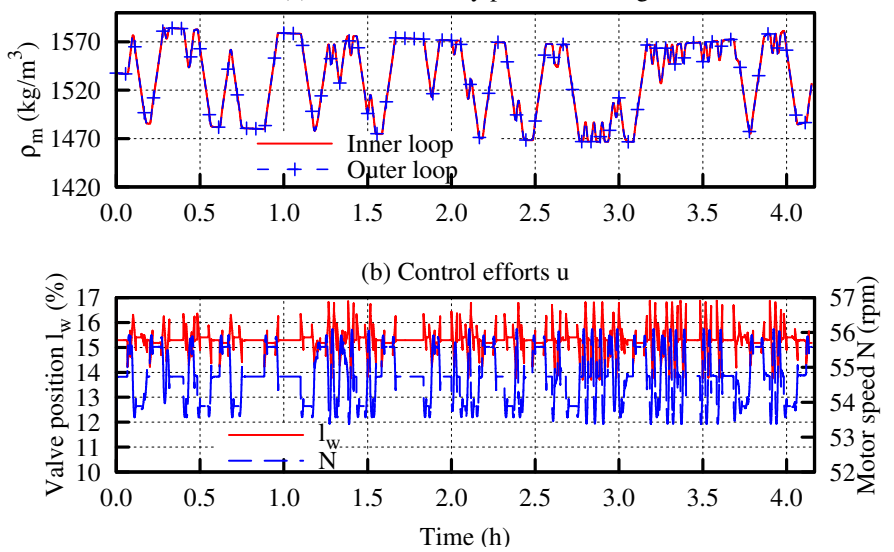

Fig. 4: Performance of the inner loop control

can conclude from the figure that the DCLC is superior to the benchmark controller in improving the fine coal quality. While the coal quality deviates from the set level during the period without feedback measurement, the DCLC is able to correct this deviation whenever new measurement is received.

Fig. 4 is presented to demonstrate the effectiveness of the inner loop controller. The first subplot reveals that the inner loop is capable of tracking the medium profile set by the outer loop control with high accuracy.

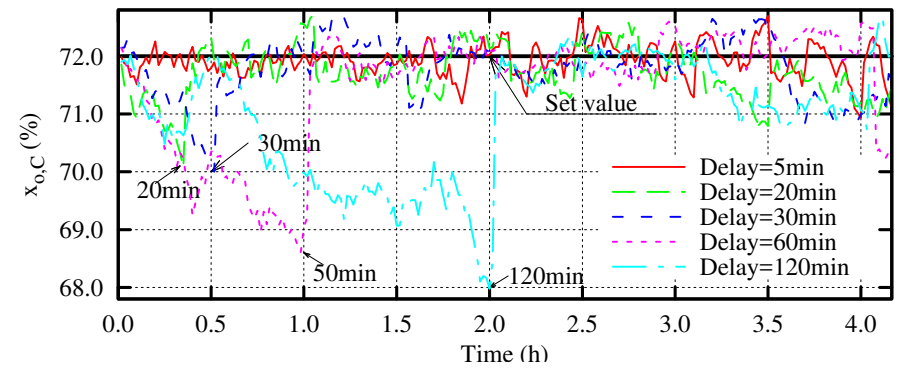

Fig. 5: Performance comparison under varying delays

2) Impacts of the measurement delay: Fig. 5 gives a visual demonstration of the performance of the DCLC under measurement delays together with the disturbances. It can be seen that before the first feedback measurement is received by the DCLC, the DMC process follows the open loop feed forward control (the benchmark). After that, the DCLC corrects the carbon content deviation according to the feedback measurement. The daily energy consumption of the DMC modules controlled by the DCLC averages at $4082.72 \mathrm{kWh}$, which is about half of that of the benchmark control (8172.18 $\mathrm{kWh}$ ). It is clear that, in general, the shorter the delay is, the better the performance indicators are.

\section{CONCLUSION}

Advantages of the controller designed in this study over existing control methods are threefold. Firstly, the controller presented is capable of making use of both feed forward and feedback measurements to optimally control the operation of the process, combining the power of an open loop feed forward 
control and a closed-loop feedback control. Secondly, the controller is designed considering a pumped-storage system in order to reduce energy consumption/cost of the plant, enabling it to improve energy efficiency and separation efficiency of the dense medium coal beneficiation process simultaneously. Lastly, the controller designed consists of two loops with each loop dedicated to its own functionality. Results obtained from a case study verify that the controller presented is superior to the benchmark control in terms of improving both the process's separation efficiency and energy efficiency.

\section{REFERENCES}

[1] U.S. Energy Information Administration, "Annual energy outlook 2015," U.S. Department of Energy, Tech. Rep. DOE/EIA-0383(2015), 2015.

[2] J. Chen, K. Chu, R. Zou, A. Yu, and A. Vince, "Prediction of the performance of dense medium cyclones in coal preparation," Minerals Engineering, vol. 31, pp. 59 - 70, 2012.

[3] L. Zhang, X. Xia, and J. Zhang, "Medium density control for coal washing dense medium cyclone circuits," IEEE Transactions on Control Systems Technology, vol. 23, no. 3, pp. 1117-1122, May 2015.

[4] K. Gordon. (2013) Can coal ash be accurately analyzed using PGNAA? [Online]. Available: http://acceleratingscience.com/mining/can-coal-ashcan-be-accurately-analyzed-using-pgnaa/

[5] L. Zhang and X. Xia, "A model predictive control for coal beneficiation dense medium cyclones," in The 19th International Federation of Automatic Control, Cape Town, South Africa, August 2014, pp. 9810 9815.

[6] L. Zhang, X. Xia, and B. Zhu, "Magnetite and water addition control for a dense medium coal washing circuit," in Chinese Automation Congress, 2015, Wuhan, China, 27-29 Nov 2015, pp. 1744-1749.

[7] L. Zhang, X. Xia, and J. Zhang, "Improving energy efficiency of cyclone circuits in coal beneficiation plants by pump-storage systems," Applied Energy, vol. 119, pp. 306 - 313, 2014.

[8] A. J. van Staden, J. Zhang, and X. Xia, "A model predictive control strategy for load shifting in a water pumping scheme with maximum demand charges," Applied Energy, vol. 88, no. 12, pp. 4785 - 4794, 2011.

[9] X. Zhuan and X. Xia, "Development of efficient model predictive control strategy for cost-optimal operation of a water pumping station," IEEE Transactions on Control Systems Technology, vol. 21, no. 4, pp. 14491454,2013

[10] L. Zhang and X. Zhuan, "Optimal operation of heavy-haul trains equipped with electronically controlled pneumatic brake systems using model predictive control methodology," IEEE Transactions on Control Systems Technology, vol. 22, no. 1, pp. 13-22, 2014.

[11] L. Zhang and X. Zhuan, "Development of an optimal operation approach in mpc framework for heavy haul trains," IEEE Transactions on Intelligent Transportation Systems, vol. 16, no. 3, pp. 1391-1400, 2015.

[12] X. Xia and J. Zhang, "Modeling and control of heavy-haul trains [applications of control]," IEEE Control Systems, vol. 31, no. 4, pp. $18-31,2011$.

[13] X. Zhuan and X. Xia, "Optimal scheduling and control of heavy haul trains equipped with electronically controlled pneumatic braking systems," IEEE Transactions on Control Systems Technology, vol. 15, no. 6, pp. 1159-1166, 2007.

[14] X. Zhuan and X. Xia, "Cruise control scheduling of heavy haul trains," IEEE Transactions on Control Systems Technology, vol. 14, no. 4, pp. 757-766, 2006.

[15] X. Zhuan and X. Xia, "Speed regulation with measured output feedback in the control of heavy haul trains," Automatica, vol. 44, pp. 242-247, 2008.

[16] B. Zhu, H. Tazvinga, and X. Xia, "Switched model predictive control for energy dispatching of a photovoltaic-diesel-battery hybrid power system," IEEE Transactions on Control Systems Technology, vol. 23, no. 3, pp. 1229-1236, 2015.

[17] H. Tazvinga, B. Zhu, and X. Xia, "Optimal power flow management for distributed energy resources with batteries," Energy Conversion and Management, vol. 102, pp. 104-110, 2015.

[18] H. Tazvinga, B. Zhu, and X. Xia, "Energy dispatch strategy for a photovoltaic-wind-diesel-battery hybrid power system," Solar Energy, vol. 108 , pp. 412-420, 2014.

[19] S. Zhang and X. Xia, "Optimal control of operation efficiency of belt conveyor systems," Applied Energy, vol. 87, no. 6, pp. 1929-1937, 2010.
[20] S. Zhang and X. Xia, "Modeling and energy efficiency optimization of belt conveyors," Applied Energy, vol. 88, no. 9, pp. 3061-3071, 2011.

[21] Y. Shen and X. Xia, "Adaptive parameter estimation for an energy model of belt conveyor with DC motor," Asian Journal of Control, vol. 16, no. 4, pp. 1122-1132, 2014.

[22] W. Badenhorst, J. Zhang, and X. Xia, "Optimal hoist scheduling of a deep level mine twin rock winder system for demand side management," Electric Power Systems Research, vol. 81, no. 5, pp. 1088-1095, 2011.

[23] B. Numbi and X. Xia, "Optimal energy control of a crushing process based on vertical shaft impactor," Applied Energy, vol. 162, pp. 16531661,2016

[24] B. Numbi and X. Xia, "Systems optimization model for energy management of a parallel HPGR crushing process," Applied Energy, vol. 149, pp. 133-147, 2015.

[25] B. Numbi, J. Zhang, and X. Xia, "Optimal energy management for a jaw crushing process in deep mines," Energy, vol. 68, pp. 337-348, 2014.

[26] A. Chatterjee, L. Zhang, and X. Xia, "Optimization of mine ventilation fan speeds according to ventilation on demand and time of use tariff," Applied Energy, vol. 146, pp. 65-73, 2015.

[27] C. Garca, D. Prett, and M. Morari, "Model predictive control: Theory and practice-a survey," Automatica, vol. 25, no. 3, pp. 335-348, 1989.

[28] L. Grüne and J. Pannek, Nonlinear Model Predictive Control: Theory and Algorithms. Springer-Verlag London Limited, 2011.

[29] X. Xia and J. Zhang, "Operation efficiency optimisation modelling and application of model predictive control," IEEE/CAA Journal of Automatica Sinica, vol. 2, no. 2, pp. 166-172, 2015.

[30] X. Xia and L. Zhang, "Industrial energy systems in view of energy efficiency and operation control," Annual Reviews in Control, 2016, In Press.

[31] X. Zhuan and X. Xia, "Development of efficient model predictive control strategy for cost-optimal operation of a water pumping station," IEEE Transactions on Control Systems Technology, vol. 21, no. 4, pp. 14491454, 2013.

[32] X. Xia, J. Zhang, and A. Elaiw, "An application of model predictive control to the dynamic economic dispatch of power generation," Control Engineering Practice, vol. 19, no. 6, pp. 638 - 648, 2011.

[33] A. Elaiw, X. Xia, and A. Shehata, "Application of model predictive control to optimal dynamic dispatch of generation with emission limitations," Electric Power Systems Research, vol. 84, no. 1, pp. 31 - 44, 2012.

[34] T. England, P. E. Hand, D. C. Michael, L. M. Falcon, and A. D. Yell, Coal Preparation in South Africa, 4th ed. Natal Witness Commercial Printers,Pietermaritzburg, South Africa, 2002.

[35] E. Meyer and I. Craig, "The development of dynamic models for a dense medium separation circuit in coal beneficiation," Minerals Engineering, vol. 23 , no. 10, pp. $791-805,2010$.

[36] T. Napier-Munn, "Modelling and simulating dense medium separation processes: A progress report," Minerals Engineering, vol. 4, no. 3C4. pp. 329 - 346, 1991.

[37] R. Sripriya, A. Dutta, P. Dhall, M. Narasimha, V. Kumar, and B. Tiwari, "An analysis of medium losses in coal washing plants," International Journal of Mineral Processing, vol. 80, no. 24, pp. 177 - 188, 2006.

[38] K. A. Atkinson, An Introduction to Numerical Analysis, 2nd ed. New York: John Wiley \& Sons, 1989.

[39] L. Hunt, R. Su, and G. Meyer, "Global transformations of nonlinear systems," IEEE Transactions on Automatic Control, vol. 28, no. 1, pp. 24-31, 1983.

[40] X. Xia, "Parameterization of decoupling control laws for affine nonlinear systems," IEEE Transactions on Automatic Control, vol. 38, no. 6, pp. 916-928, 1993.

[41] H. Chen and F. Allgower, "A quasi-infinite horizon nonlinear model predictive control scheme with guaranteed stability," Automatica, vol. 34, no. 10 , pp. $1205-1217,1998$

[42] A. R. Teel, L. Moreau, and D. Nesic, "A unified framework for inputto-state stability in systems with two time scales," IEEE Transactions on Automatic Control, vol. 48, no. 9, pp. 1526-1544, 2003.

[43] H. K. Khalil, Nonlinear Systems, 3rd ed., A. Dworkin, Ed. Prentice Hall, 2002.

[44] D. Mayne, J. Rawlings, C. Rao, and P. Scokaert, "Constrained model predictive control: Stability and optimality," Automatica, vol. 36, no. 6 , pp. 789-814, 2000 .

[45] X. Xia and J. Zhang, "Mathematical description for the measurement and verification of energy efficiency improvement," Applied Energy, vol. 111, pp. $247-256,2013$. 